\title{
Chromosome Painting Does Not Support a Sex Chromosome Turnover in Lacerta agilis Linnaeus, 1758
}

\author{
Artem P. Lisachov ${ }^{a}$ Massimo Giovannotti ${ }^{b} \quad$ Jorge C. Pereirac \\ Daria A. Andreyushkovad Svetlana A. Romanenko d, e \\ Malcolm A. Ferguson-Smith ${ }^{c}$ Pavel M. Borodin ${ }^{a}$ e Vladimir A. Trifonov ${ }^{d, e}$ \\ a Institute of Cytology and Genetics SB RAS, Novosibirsk, Russia; ${ }^{\text {b }}$ Dipartimento di Scienze della Vita e dell'Ambiente, \\ Università Politecnica delle Marche, Ancona, Italy; ${ }^{C}$ Department of Veterinary Medicine, Cambridge Resource Centre \\ for Comparative Genomics, Cambridge, UK; ${ }^{\mathrm{d}}$ Institute of Molecular and Cellular Biology SB RAS, Novosibirsk, Russia; \\ ${ }^{e}$ Novosibirsk State University, Novosibirsk, Russia
}

\section{Keywords}

Lacertidae $\cdot$ Reptilia $\cdot$ Sex chromosomes $\cdot$ Sex determination · Zoo-FISH

\begin{abstract}
Reptiles show a remarkable diversity of sex determination mechanisms and sex chromosome systems, derived from different autosomal pairs. The origin of the ZW sex chromosomes of Lacerta agilis, a widespread Eurasian lizard species, is a matter of discussion: is it a small macrochromosome from the 11-18 group common to all lacertids, or does this species have a unique ZW pair derived from the large chromosome 5? Using independent molecular cytogenetic methods, we investigated the karyotype of L. agilis exigua from Siberia, Russia, to identify the sex chromosomes. FISH with a flow-sorted chromosome painting probe derived from $L$. strigata and specific to chromosomes 13, 14, and Z confirmed that the $Z$ chromosome of $L$. agilis is a small macrochromosome, the same as in L. strigata. FISH with the telomeric probe showed an extensive accumulation of the telo-
\end{abstract}

mere-like repeat in the $\mathrm{W}$ chromosome in agreement with previous studies, excluding the possibility that the lineages of $L$. agilis studied in different works could have different sex chromosome systems due to a putative intra-species polymorphism. Our results reinforce the idea of the stability of the sex chromosomes and lack of evidence for sex-chromosome turnovers in known species of Lacertidae.

(c) 2020 S. Karger AG, Basel

Reptiles have diverse mechanisms of sex determination. In some taxa, such as crocodiles, red-eared turtles, and leopard geckos, the sex of the offspring is determined by the temperature of egg incubation, and there are no specific sex-linked genes or chromosomes [Viets et al., 1993]. In other lineages, different chromosomes independently acquired sex-determining genes (via mutations of their original genes or translocations of genes from other chromosomes) and became sex chromosomes of either $\mathrm{XX} / \mathrm{XY}$ or ZZ/ZW type [Pokorná and Kratochvíl, 2009]. The most studied reptilian sex chromosomes are those of
Artem P. Lisachov

Laboratory of Recombinational and Segregational Analysis Institute of Cytology and Genetics SB RAS

Lavrentyeva av. 10, Novosibirsk 630090 (Russia)

lisachev@bionet.nsc.ru 
pleurodont iguanas (XX/XY) [Alföldi et al., 2011; Gamble et al., 2014; Rovatsos et al., 2014; Kichigin et al., 2016; Giovannotti et al., 2017b; Lisachov et al., 2019], advanced snakes (ZZ/ZW) [Matsubara et al., 2006; Vicoso et al., 2013; Rovatsos et al., 2015], and anguimorphs (ZZ/ZW) [Matsubara et al., 2014; Rovatsos et al., 2019b]. In certain cases, sex chromosome systems are not universal for such large clades, but are specific to one species or a group of related species. This is a rather common situation in geckos [Gamble, 2010].

For all reptile sex chromosome systems, the exact master sex-determining genes and therefore the molecular mechanisms underlying sex determination are unknown. Uncovering these mechanisms will advance the understanding of general principles of the evolution of sex determination. It may also help to predict the sensitivity of sex chromosome systems to climate change. Extreme temperatures can override chromosomal sex determination in some squamate reptiles (described for Agamidae and Scincidae), skew the sex ratio, and thus make their populations unstable and prone to extinction [Holleley et al., 2015].

The first step in finding the master sex-determining genes is uncovering the correspondence between a certain reptile sex chromosome system and the homologous chromosome or syntenic block in the reference sauropsid genomes, such as chicken (Gallus gallus, GGA) and anole lizard (Anolis carolinensis, ACA). For example, the XY chromosomes of $A$. carolinensis correspond to chicken chromosome 15 (GGA15) [Alföldi et al., 2011]. This "genomic identity" is known for many reptile sex chromosome systems and can be uncovered by different molecular genetic, genomic, and cytogenetic approaches [Deakin and Ezaz, 2019].

However, for some taxa different works and methods produced contradicting results. This can be due to intraspecific polymorphisms, wrong species identifications, or technical errors [discussed by Gamble, 2010]. The sand lizard (Lacerta agilis, LAG), a common Eurasian species, is one of the examples of such contradiction. It belongs to the family Lacertidae, which is long known to have a ZW sex chromosome system [Ivanov and Fedorova, 1970].

The lacertid chromosomes generally share the same acrocentric morphology and gradually decrease in length; therefore, individual chromosomes are difficult to distinguish. Although the $\mathrm{W}$ chromosome can be identified by heterochromatinization and repetitive sequence accumulation [Capriglione et al., 1994], the $\mathrm{Z}$ chromosome is "hidden" among the autosomes, and its genetic content and even the position in the karyotype remained unknown for a long time.

Srikulnath et al. [2014] identified chromosome 5 of L. agilis from Sweden (L. agilis agilis) (LAG5) as the putative $\mathrm{Z}$ chromosome using Hoechst staining. By FISH mapping of cDNA probes of protein-coding genes, they found that this chromosome is a homologue of the short arm of the $A$. carolinensis chromosome 3 (ACA3p) and of the chicken chromosomes 6 and 9 (GGA6, GGA9). In another work, Matsubara et al. [2015] described the $\mathrm{W}$ chromosome of these lizards and found that it contains $\mathrm{C}$-positive heterochromatin, enriched with telomere-like sequences.

Later, Rovatsos et al. [2016, 2019c] used transcriptome analysis and qPCR to identify hemizygous, and thus suggestively Z-linked, genes in several species of lacertids, including L. agilis from the Czech Republic (L. agilis argus). In these studies, the genes reported as Z-specific by Srikulnath et al. [2014] were identified as (pseudo)autosomal. The orthologues of the Z-linked genes which were determined by Rovatsos et al. [2016, 2019c] are located in 2 microchromosomes of $A$. carolinensis, ACA11 and ACA16 (homologous to GGA4p and GGA17, respectively) [Kichigin et al., 2016]. Rovatsos et al. suggested that the $\mathrm{Z}$ chromosome of lacertids should be one of the small macrochromosomes, formed via fusion of 2 ancestral squamate microchromosomes [Uno et al., 2012]. Their results were supported by the genome sequencing project of Podarcis muralis [Andrade et al., 2019].

There are 2 possible explanations for this contradiction. First, it is possible that the different lineages of $L$. agilis, studied in these 2 works, indeed have different sex chromosome systems. Namely, the lineage examined by Srikulnath et al. [2014] might have experienced a sex chromosome turnover, leading to the loss of the original lacertid sex chromosome system and appearance of a new system, based on another chromosome pair. The cases of such turnovers, when a taxon "forsakes" a well-established sex chromosome system and acquires a new one, are rather rare [Johnson Pokorná and Kratochvíl, 2016], but known in lizards [Nielsen et al., 2019] and even in mammals [Matveevsky et al., 2017]. An intraspecific polymorphism in sex-determining systems is also possible, as in the case of the frog Glandirana rugosa [Ogata et al., 2018]. The second possibility is that one or both these identifications of the L. agilis sex chromosome are erroneous.

No additional studies with independent methods were conducted so far to investigate this issue. Meanwhile, resolving this contradiction is crucial for understanding the 
patterns of evolution of sex-determining mechanisms in reptiles in general. If confirmed, "the curious case of $L a$ certa agilis" could become an interesting model to study the processes of sex determination evolution, since the sex chromosomes of this species might represent a young sex chromosome system with a known time of origin, based on the established phylogenetic tree of Lacertidae [Garcia-Porta et al., 2019], and with an autosomal homologue in the genomes of closely related species [Andrade et al., 2019].

Our aim was to resolve the question regarding the identification of 2 different sex chromosome systems in L. agilis applying methods which were not used previously. In this work, we investigated the karyotype of $L$. agilis exigua from Novosibirsk (Russia) to identify and describe its $\mathrm{Z}$ and $\mathrm{W}$ chromosomes. The $\mathrm{Z}$ chromosome was identified by FISH using a flow-sorted chromosome painting probe derived from L. strigata, containing its chromosomes 13, 14, and Z. Sorted chromosome probes of lacertids were generated, and chromosome painting was conducted previously, but with limited success, and L. agilis and L. strigata were not studied [Rojo Oróns, 2015]. The $\mathrm{W}$ chromosome was identified by its size and by FISH with a telomere-specific probe.

\section{Materials and Methods}

The fibroblast cell culture of L. strigata, here used for flowsorting, was established previously by Giovannotti et al. [2018]. The flow-sorted chromosome libraries were obtained using a Mo$\mathrm{Flo}^{\circledR}$ (Beckman Coulter) high-speed cell sorter at the Cambridge Resource Centre for Comparative Genomics, Department of Veterinary Medicine, University of Cambridge, Cambridge, UK, as described previously [Yang et al., 1995]. The painting probes were generated from the DOP-PCR amplified libraries by incorporation of Flu-12-dUTP (Bioron, Germany) during a secondary DOPPCR [Telenius et al., 1992].

The fibroblast cell culture of $L$. agilis was obtained from muscle tissues of 1 juvenile female $L$. a. exigua, originating from Novosibirsk province (Russia). Cell cultures were established in the Laboratory of Animal Cytogenetics, the Institute of Molecular and Cellular Biology, Russia, using enzymatic treatment of tissues as described previously [Stanyon and Galleni, 1991; Romanenko et al., 2015]. All cell lines were deposited in the IMCB SB RAS cell bank ("The general collection of cell cultures", 0310-2016-0002). Metaphase chromosome spreads were prepared from chromosome suspensions obtained from early passages of primary fibroblast cultures as described previously [Yang et al., 1999; Graphodatsky et al., 2000, 2001].

The telomere-specific probe was generated by PCR with selfannealing oligonucleotide primers, as described previously [Ijdo et al., 1991], and labeled with TAMRA-dUTP (Biosan, Novosibirsk, Russia) in a secondary PCR. FISH was performed following standard techniques [Liehr et al., 2017]. The slides were treated with

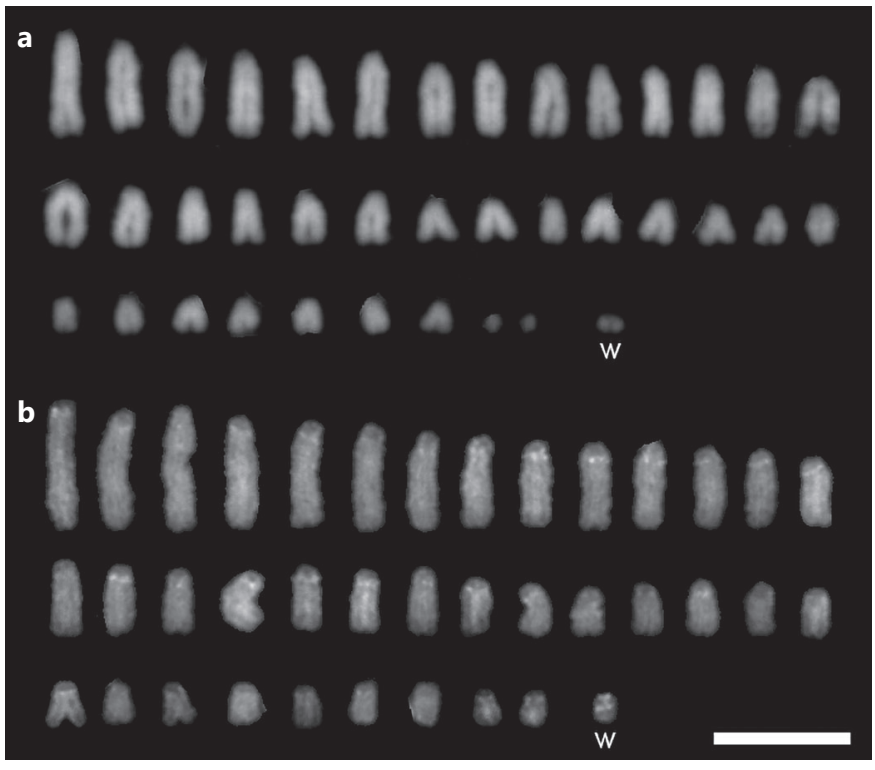

Fig. 1. Karyotypes of Lacerta strigata (a) and L. agilis (b). DAPI staining. Scale bar, $10 \mu \mathrm{m}$.

RNAse A solution $(0.2 \mathrm{mg} / \mathrm{mL})$ in $2 \times \mathrm{SSC}$ at $37^{\circ} \mathrm{C}$ for $30 \mathrm{~min}$. Then the slides were washed in $2 \times$ SSC for $5 \mathrm{~min}$, and treated with trypsin solution $(0.12 \%)$ for $15 \mathrm{~s}$. After the subsequent washes, the slides were denatured in $70 \%$ formamide, $2 \times$ SSC at $70^{\circ} \mathrm{C}$ for 3 min. The hybridization mix contained $16 \mu \mathrm{L}$ of $50 \%$ formamide, $10 \%$ sodium dextran sulfate, $0.2 \%$ Tween 20 in $2 \times$ SSC, and $0.2 \mu \mathrm{g}$ of the probe. The hybridization mix was denatured at $96^{\circ} \mathrm{C}$ for 5 min and then incubated at $37^{\circ} \mathrm{C}$ for $1 \mathrm{~h}$. Thereafter, it was applied to the slides. The hybridization lasted $12 \mathrm{~h}$ for the telomeric probe and for same-species chromosome painting, and $48 \mathrm{~h}$ for crossspecies chromosome painting. The preparations were analyzed with an Axioplan 2 Imaging microscope (Carl Zeiss) equipped with a CCD camera (CV M300, JAI), CHROMA filter sets, and the ISIS4 image processing package (MetaSystems $\mathrm{GmbH}$ ). The brightness and contrast of all images were enhanced using Corel PaintShop Photo Pro X6 (Corel Corp).

\section{Results}

The karyotype of L. strigata has 38 chromosomes with a heteromorphic W chromosome (Fig. 1a). Here, for the first time, we have obtained flow-sorted chromosome libraries of a Lacerta species. The sorted karyotype of $L$. strigata comprised 17 peaks (LST_A to LST_Q) (Fig. 2). The probe derived from peak LST_L was found to hybridize to 5 chromosomes on the metaphases of the specimen used for establishing the fibroblast cell culture. Therefore, it was concluded that this peak contained 2 autosome pairs and the $\mathrm{Z}$ chromosome (Fig. 3a). The autosomes 


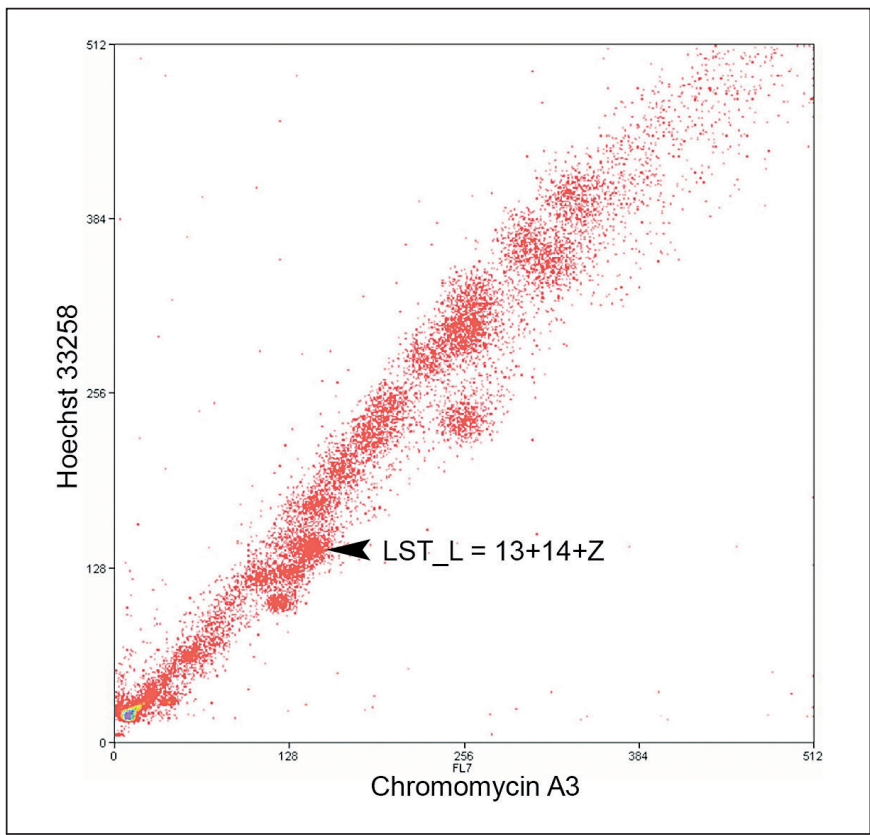

Fig. 2. Flow-sorted karyotype of Lacerta strigata. The $x$ and $y$ axes report fluorescence intensity for each fluorochrome. The peak LST_L is indicated by an arrowhead.

were identified as pairs 13 and 14 based on the position of the LST_L peak in the flow karyotype. The co-occurrence of several chromosomes in the same probe is due to their very similar size and GC-content, which did not allow their separation during chromosome sorting.

The karyotype of L. agilis also has 38 chromosomes, including the heteromorphic $\mathrm{W}$ chromosome, the smallest element of the karyotype (Fig. 1b). FISH with the LST_L probe showed hybridization with 5 small macrochromosomes, the same as in the hybridization with $L$. strigata (Fig. 3b). FISH with the telomeric probe showed the accumulation of the telomeric repeat in the $\mathrm{W}$ chromosome (Fig. 4).

\section{Discussion}

Our results do not confirm LAG5 as a sex chromosome. This resolves the 5-year-long debate concerning the sex chromosomes of the sand lizard.

The karyotypes of the studied Lacerta species match with previous descriptions [Ivanov and Fedorova, 1970; De Smet, 1981]. The absence of a signal on the W chromosome in FISH experiments with the Z-derived flowsorted probe is apparently due to the fact that the W chro-
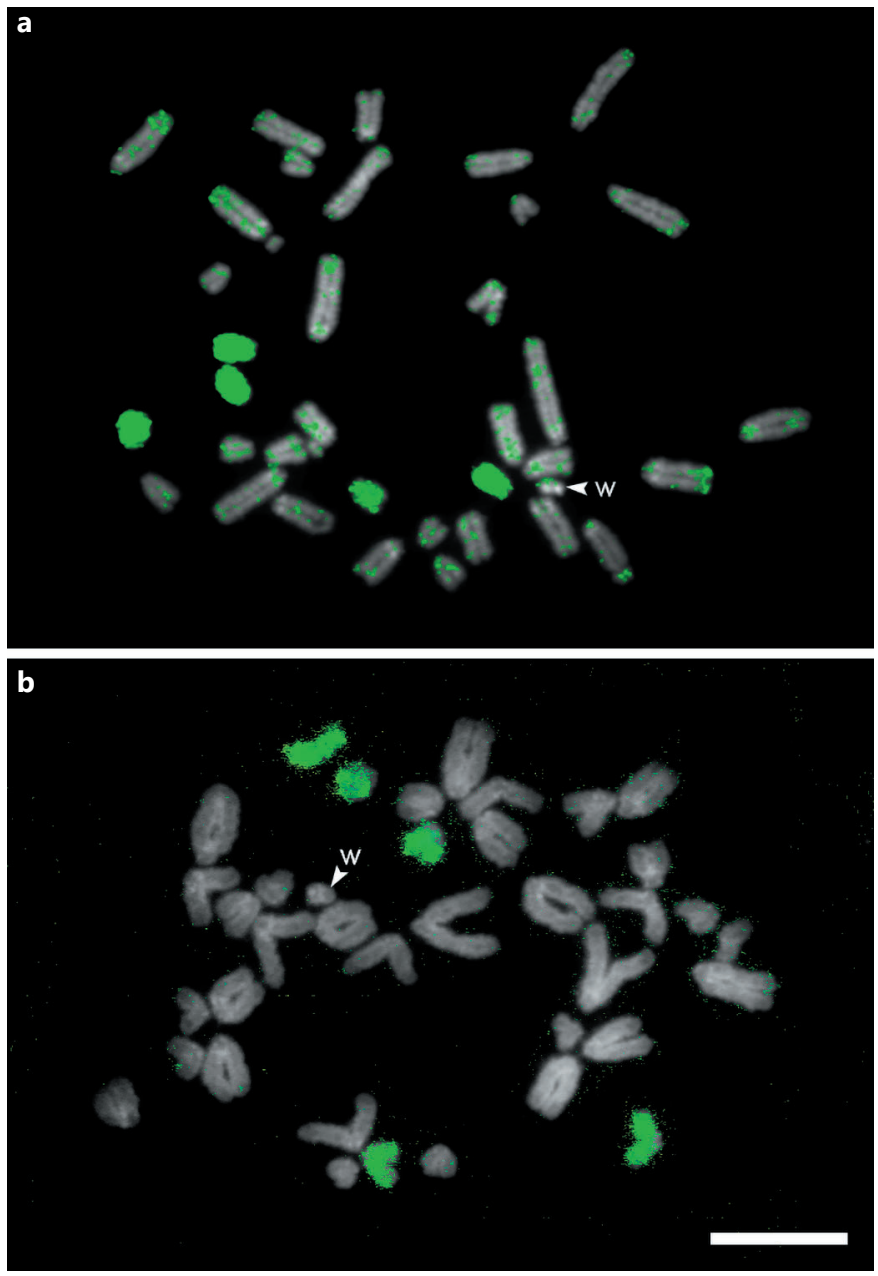

Fig. 3. FISH with the LST_L probe (green) on Lacerta strigata (a) and L. agilis (b) metaphase plates. Scale bar, $10 \mu \mathrm{m}$.

mosome of lacertids is too degenerated and does not hybridize with the DNA from the $\mathrm{Z}$ chromosome [Andrade et al., 2019; Rovatsos et al., 2019c].

The fact that the LST_L probe painted 5 chromosomes in L. agilis as well as in L. strigata indicates that these 2 species share the same sex chromosome system. If $L$. agilis had experienced a hypothetical sex chromosome turnover, leading to the formation of neo-sex chromosomes derived from LAG5, and the original lacertid $\mathrm{Z}$ chromosome had returned to the autosomal state (as in the sex chromosome turnover in the lineage of the genus Paroedura, Gekkonidae, which includes P. picta and several other species [Koubová et al., 2014; Rovatsos et al., 2019a]), the LST_L probe would have hybridized with 6 chromosomes in L. agilis (3 homologous pairs). The hy- 


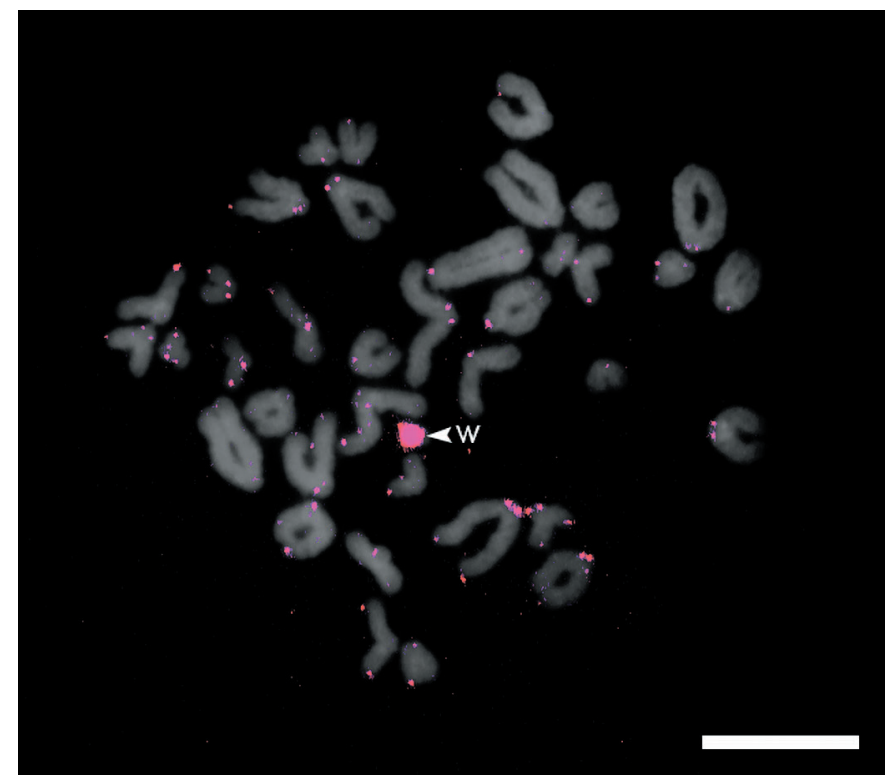

Fig. 4. FISH with the telomeric probe (red) on a Lacerta agilis metaphase plate. Scale bar, $10 \mu \mathrm{m}$.

bridization of the LST_L probe with 5 chromosomes was observed in multiple complete metaphases, which confirms the reliability of our results.

The possibility that these 2 species share the same sex chromosome turnover is excluded by the size of the chromosomes which constitute the LST_L probe: they belong to the fraction of small chromosomes, whereas LAG5/ LST5 are nearly twice as large. The small size of the Z chromosome in L. agilis and L. strigata fits well with the results of Rovatsos et al. [2016, 2019c] and Andrade et al. [2019], which showed that the lacertid $\mathrm{Z}$ originates from the fusion of 2 microchromosomes of ancestral squamates.

There could be still a possibility that the sex chromosome turnover is not universal for L. agilis, but is specific to the lineage studied by Srikulnath et al. [2014] and Matsubara et al. [2015]: the nominative subspecies L. a. agilis. However, the features of the $\mathrm{W}$ chromosome of the lizards which were studied in these works provide evidence against this explanation.

First, in case of such a recent turnover, the $\mathrm{Z}$ and $\mathrm{W}$ chromosomes would be expected to be homomorphic. The sand lizards from the Czech Republic, for which the retention of the ancestral sex chromosome system has been shown by Rovatsos et al. [2016], and the sand lizards from Sweden are very closely related: molecular phylogenetic analysis based on mtDNA markers showed that the traditionally recognized subspecies $L$. $a$. argus and $L$. $a$. agilis form 1 clade and are not monophyletic in relation to each other [Andres et al., 2014]. However, the W chromosome in L. a. agilis is highly divergent from the putative $\mathrm{Z}$ chromosome [Srikulnath et al., 2014; Matsubara et al., 2015].

Second, the relative size and telomeric repeat accumulation of the L. a. agilis W chromosome [Matsubara et al., $2015]$ correspond well with the features of the $\mathrm{W}$ chromosome of $L$. a. exigua that were observed in this study. Furthermore, $\mathrm{W}$ chromosomes of similar size and repeat content are characteristic for other species of Lacerta: all studied Lacerta species including L. agilis have telomeric repeat accumulation in the $\mathrm{W}$ chromosome, and share a monophyletic W-specific subfamily of the IMO-TaqI satellite DNA [Giovannotti et al., 2018]. Considering the fact that the sequence content of the $\mathrm{W}$ chromosomes in the related genera Iberolacerta and Timon is different from that of Lacerta, as shown by CGH [Rojo Oróns, 2015], and that the $\mathrm{W}$-specific repetitive DNA can also differ in different populations of the same species [Giovannotti et al., 2017a], the sharing of the same repetitive DNAs by the $\mathrm{W}$ chromosomes of different Lacerta species seems to indicate stability and homology for the ZZ/ZW system within this genus.

Thus, we conclude that the sex chromosomes of $L$. agilis specimens studied by Srikulnath et al. [2014] are most probably not different from the sex chromosomes of $L$. agilis individuals studied in the current work. Therefore, it is unlikely that LAG5 is the Z chromosome in this species. Recently, Rovatsos et al. [2019c] doubled the number of lacertid species included in their analysis, and found no confirmation for several other putative cases of sex chromosome turnovers in some lacertids. Our results agree with this conclusion and reinforce the idea of stability of the lacertid ZZ/ZW sex chromosome system.

The library of flow-sorted chromosome probes of $L$. strigata is a powerful tool to study chromosome evolution in Lacertidae. It may be further used to test the sex chromosome homology and identify chromosomal rearrangements in other species of lacertids, which were previously not studied using molecular cytogenetic methods.

\section{Acknowledgement}

We thank S.O. Baturin and A.I. Stekleneva (Institute of Cytology and Genetics) for help in obtaining the specimen of L. a. exigua. We thank the Microscopic Center of the Siberian Branch of the Russian Academy of Sciences for granting access to microscopic equipment. 


\section{Statement of Ethics}

All manipulations with animals were approved by the Institute of Molecular and Cellular Biology Ethics Committee (statement \#01/18 from March 5, 2018).

\section{Disclosure Statement}

The authors have no conflicts of interest to declare.

\section{Funding Sources}

This work was supported by the research grant \#18-34-00182 from the Russian Foundation for Basic Research, the research grant \#19-14-00050 from the Russian Science Foundation, the re- search grant \#AAAA-A17-117071240065-4 from the Ministry of Science and Higher Education (Russia) via the Institute of Cytology and Genetics, and the grant No. I36C18004900005 awarded to Massimo Giovannotti from Università Politecnica delle Marche.

\section{Author Contributions}

A.P.L., V.A.T., and P.M.B. designed the study. A.P.L. produced the figures and wrote the initial draft of the manuscript. M.G. and S.A.R. prepared the cell cultures. J.C.P. and M.A.F.S. performed flow sorting. J.C.P. and D.A.A. performed FISH. All authors participated in writing and editing the manuscript.

\section{References}

-Alföldi J, Di Palma F, Grabherr M, Williams C, Kong $L$, et al: The genome of the green anole lizard and a comparative analysis with birds and mammals. Nature 477:587-591 (2011).

-Andrade P, Pinho C, Pérez i de Lanuza GP, Afonso $\mathrm{S}$, Brejcha J, et al: Regulatory changes in pterin and carotenoid genes underlie balanced color polymorphisms in the wall lizard. Proc Natl Acad Sci USA 116:5633-5642 (2019).

-Andres C, Franke F, Bleidorn C, Bernhard D, Schlegel M: Phylogenetic analysis of the Lacerta agilis subspecies complex. Syst Biodivers 12:43-54 (2014).

-Capriglione T, Olmo E, Odierna G, Kupriyanova LA: Mechanisms of differentiation in the sex chromosomes of some Lacertidae. AmphibiaReptilia 15:1-8 (1994).

Deakin JE, Ezaz T: Understanding the evolution of reptile chromosomes through applications of combined cytogenetics and genomics approaches. Cytogenet Genome Res 157:7-20 (2019).

De Smet WHO: Description of the orcein stained karyotypes of 36 lizard species (Lacertilia, Reptilia) belonging to the families Teiidae, Scincidae, Lacertidae, Cordylidae and Varanidae (Autarchoglossa). Acta Zool Pathol Antverpiensia 76:73-118 (1981).

-Gamble T: A review of sex determining mechanisms in geckos (Gekkota: Squamata). Sex Dev 4:88-103 (2010).

- Gamble T, Geneva AJ, Glor RE, Zarkower D: Anolis sex chromosomes are derived from a single ancestral pair. Evolution 68:10271041 (2014).

Garcia-Porta J, Irisarri I, Kirchner M, Rodríguez A, Kirchhof S, et al: Environmental temperatures shape thermal physiology as well as diversification and genome-wide substitution rates in lizards. Nat Commun 10:4077 (2019).
Giovannotti M, Nisi Cerioni P, Slimani T, Splen- Johnson Pokorná M, Kratochvíl L: What was the diani A, Paoletti A, et al: Cytogenetic characterization of a population of Acanthodactylus lineomaculatus Duméril and Bibron, 1839 (Reptilia, Lacertidae) from South-western Morocco and insights into sex chromosome evolution. Cytogenet Genome Res 153:86-95 (2017a).

Giovannotti M, Trifonov VA, Paoletti A, Kichigin IG, O'Brien PCM, et al: New insights into sex chromosome evolution in anole lizards (Reptilia, Dactyloidae). Chromosoma 126:245260 (2017b).

- Giovannotti M, Nisi Cerioni P, Rojo V, Olmo E, Slimani T, et al: Characterization of a satellite DNA in the genera Lacerta and Timon (Reptilia, Lacertidae) and its role in the differentiation of the W chromosome. J Exp Zool B Mol Dev Evol 330:83-95 (2018).

Graphodatsky AS, Sablina OV, Meyer MN, Malikov VG, Isakova EA, et al: Comparative cytogenetics of hamsters of the genus Calomyscus. Cytogenet Cell Genet 88:296-304 (2000).

Graphodatsky AS, Yang F, O'Brien PC, Perelman P, Milne BS, et al: Phylogenetic implications of the 38 putative ancestral chromosome segments for four canid species. Cytogenet Cell Genet 92:243-247 (2001).

-Holleley CE, O’Meally D, Sarre, SD, Graves JAM, Ezaz T, et al: Sex reversal triggers the rapid transition from genetic to temperature-dependent sex. Nature 523:79-82 (2015).

Ijdo JW, Wells RA, Baldini A, Reeders ST: Improved telomere detection using a telomere

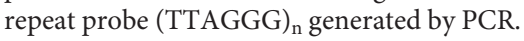
Nucleic Acids Res 19:4780 (1991).

Ivanov VG, Fedorova TA: Sex heteromorphism of chromosomes in Lacerta strigata Eichwald (in Russian). Tsitologiia 12:1582-1585 (1970). ancestral sex-determining mechanism in amniote vertebrates? Biol Rev Camb Philos Soc 91:1-12 (2016).

Kichigin IG, Giovannotti M, Makunin AI, Ng BL, Kabilov ML, et al: Evolutionary dynamics of Anolis sex chromosomes revealed by sequencing of flow sorting-derived microchromosome-specific DNA. Mol Genet Genomics 291:1955-1966 (2016).

Koubová M, Pokorná MJ, Rovatsos M, Farkačová K, Altmanová M, Kratochvíl L: Sex determination in Madagascar geckos of the genus Paroedura (Squamata: Gekkonidae): are differentiated sex chromosomes indeed so evolutionary stable? Chromosome Res 22:441-452 (2014).

Liehr T, Kreskowski K, Ziegler M, Piaszinski K, Rittscher K: The standard FISH procedure, in Liehr T (ed): Fluorescence in Situ Hybridization (FISH), pp 109-118 (Springer, Berlin 2017).

- Lisachov AP, Makunin AI, Giovannotti M, Pereira JC, Druzhkova AS, et al: Genetic content of the neo-sex chromosomes in Ctenonotus and Norops (Squamata, Dactyloidae) and degeneration of the $\mathrm{Y}$ chromosome as revealed by high-throughput sequencing of individual chromosomes. Cytogenet Genome Res 157:115-122 (2019).

Matsubara K, Tarui H, Toriba M, Yamada K, Nishida-Umehara C, et al: Evidence for different origin of sex chromosomes in snakes, birds, and mammals and step-wise differentiation of snake sex chromosomes. Proc Natl Acad Sci USA 103:18190-18195 (2006).

- Matsubara K, Sarre SD, Georges A, Matsuda Y, Graves JAM, Ezaz T: Highly differentiated ZW sex microchromosomes in the Australian Varanus species evolved through rapid amplification of repetitive sequences. PLoS One 9:e95226 (2014). 
Matsubara K, Uno Y, Srikulnath K, Matsuda Y, Miller E, Olsson M: No interstitial telomeres on autosomes but remarkable amplification of telomeric repeats on the $\mathrm{W}$ sex chromosome in the sand lizard (Lacerta agilis). J Hered 106:753-757 (2015).

-Matveevsky S, Kolomiets O, Bogdanov A, Hakhverdyan $\mathrm{M}$, Bakloushinskaya I: Chromosomal evolution in mole voles Ellobius (Cricetidae, Rodentia): bizarre sex chromosomes, variable autosomes and meiosis. Genes 8:E306 (2017).

- Nielsen SV, Guzmán-Méndez IA, Gamble T, Blumer M, Pinto BJ, et al: Escaping the evolutionary trap? Sex chromosome turnover in basilisks and related lizards (Corytophanidae: Squamata). Biol Lett 15:20190498 (2019).

- Ogata M, Lambert M, Ezaz T, Miura I: Reconstruction of female heterogamety from admixture of XX-XY and ZZ-ZW sex-chromosome systems within a frog species. Mol Ecol 27:4078-4089 (2018).

Pokorná M, Kratochvíl L: Phylogeny of sex-determining mechanisms in squamate reptiles: are sex chromosomes an evolutionary trap? Zool J Linn Soc 156:168-183 (2009).

Rojo Oróns V: Cytogenetic and molecular characterization of lacertid lizard species from the Iberian Peninsula. Thesis, Universidade da Coruña (2015).
Romanenko SA, Biltueva LS, Serdyukova NA, Kulemzina AI, Beklemisheva VR, et al: Segmental paleotetraploidy revealed in sterlet (Acipenser ruthenus) genome by chromosome painting. Mol Cytogenet 8:90 (2015).

Rovatsos M, Pokorná M, Altmanová M, Kratochvíl L: Cretaceous park of sex determination: sex chromosomes are conserved across iguanas. Biol Lett 10:20131093 (2014).

Rovatsos M, Vukić J, Lymberakis P, Kratochvíl L: Evolutionary stability of sex chromosomes in snakes. Proc Biol Sci 282:20151992 (2015).

Rovatsos M, Vukić J, Altmanová M, Johnson Pokorná M, Moravec J, Kratochvíl L: Conservation of sex chromosomes in lacertid lizards. Mol Ecol 25:3120-3126 (2016).

- Rovatsos M, Farkačová K, Altmanová M, Johnson Pokorná M, Kratochvíl L: The rise and fall of differentiated sex chromosomes in geckos. Mol Ecol 28:3042-3052 (2019a).

Rovatsos M, Rehák I, Velenský P, Kratochvíl L: Shared ancient sex chromosomes in varanids, beaded lizards, and alligator lizards. Mol Biol Evol 36:1113-1120 (2019b).

Rovatsos M, Vukić J, Mrugała A, Suwala G, Lymberakis P, Kratochvíl L: Little evidence for switches to environmental sex determination and turnover of sex chromosomes in lacertid lizards. Sci Rep 9:7832 (2019c).

Srikulnath K, Matsubara K, Uno Y, Nishida C, Olsson M, Matsuda Y: Identification of the linkage group of the $\mathrm{Z}$ sex chromosomes of the sand lizard (Lacerta agilis, Lacertidae) and elucidation of karyotype evolution in lacertid lizards. Chromosoma 123:563-575 (2014).
Stanyon R, Galleni L: A rapid fibroblast culture technique for high resolution karyotypes. Boll Zool 58:81-83 (1991).

- Telenius H, Pelmear AH, Tunnacliffe A, Carter NP, Behmel A et al: Cytogenetic analysis by chromosome painting using DOP-PCR amplified flow-sorted chromosomes. Genes Chromosomes Cancer 4:257-263 (1992).

Uno Y, Nishida C, Tarui H, Ishishita S, Takagi C, et al: Inference of the protokaryotypes of amniotes and tetrapods and the evolutionary processes of microchromosomes from comparative gene mapping. PLoS One 7:e53027 (2012).

-Vicoso B, Emerson JJ, Zektser Y, Mahajan S, Bachtrog D: Comparative sex chromosome genomics in snakes: differentiation, evolutionary strata, and lack of global dosage compensation. PLoS Biol 11:e1001643 (2013).

-Viets BE, Tousignant A, Ewert MA, Nelson CE, Crews D: Temperature-dependent sex determination in the leopard gecko, Eublepharis macularius. J Exp Zool 265:679-683 (1993).

- Yang F, Carter NP, Shi L, Ferguson-Smith MA: A comparative study of karyotypes of muntjacs by chromosome painting. Chromosoma 103: 642-652 (1995).

- Yang F, O’Brien PC, Milne BS, Graphodatsky AS, Solanky N, et al: A complete comparative chromosome map for the dog, red fox, and human and its integration with canine genetic map. Genomics 62:189-202 (1999). 